\title{
Numerical Aspects of Discrete and Continuum Hybrid Models in Cell Biology
}

J. C. Dallon

dallon@math.byu.edu

Follow this and additional works at: https://scholarsarchive.byu.edu/facpub

Part of the Mathematics Commons

\section{Original Publication Citation}

J.C. Dallon: Numerical Aspects of Discrete and Continuum Hybrid Models in Cell Biology. Applied Numerical Mathematics 32: 137-159 (2).

\section{BYU ScholarsArchive Citation}

Dallon, J. C., "Numerical Aspects of Discrete and Continuum Hybrid Models in Cell Biology" (2000).

Faculty Publications. 1103.

https://scholarsarchive.byu.edu/facpub/1103 


\title{
Numerical Aspects of Discrete and Continuum Hybrid Models in Cell Biology
}

\author{
John Dallon \\ Department of Mathematics \\ Heriot-Watt University \\ Edinburgh, EH14 4AS U.K.
}

July 12, 2000

keywords: discrete-continuum hybrid models, numerical methods, biological modeling, wound healing, dictyostelium discoideum, particle-mesh methods

\begin{abstract}
In this paper we introduce a method of modeling which mixes continuum and discrete variables, and explain two models in cell biology that use this method. The first application deals with wound healing, more specifically the collagen alignment in scar tissue formation and the second models early aggregation in the cellular slime mold Dictyostelium discoideum. We solve these models using numerical techniques similar to the particle-in-cell method which requires that the discrete and continuum variables are interpolated one to the other. The implementational and numerical details are discussed in an informal and practical manner with particular attention given to the problem of interpolation.
\end{abstract}

\section{Introduction}

Since the time of Newton, continuum mathematical models have been used to describe the behavior of what we believe to be fundamentally discrete physical systems. As a result, much time and effort has been spent in justifying these continuum formulations with methods from statistical mechanics by using the fundamental properties, including the discreteness of the system, to derive the models. In an ironic twist due to the advent of computers, these continuum models of discrete systems are frequently approximated by discrete models which can be solved numerically. As computers have become more powerful, there has been renewed interest in models maintaining a discrete formulation from the outset. 
Modeling efforts utilizing the discrete nature of the physical system vary widely and include applications in chemistry, modeling macromolecules [28] and chemically reactive systems [4]; physics, in textile research [10], underground waterflow [31] and crystalline structures [9]; and biology, modeling cellular slime mold [23], the interaction of fibroblasts [8] and cell to cell signaling [3]. The purpose of this paper is to describe a discrete and continuum hybrid method applied to biological systems. In so doing we highlight key differences, including implementational differences, with the more standard continuum modeling. The method is ideal for systems involving cell motion, and we consider two applications of this type as case studies. The first of these models the process of collagen production and alignment during wound healing [6] and the second deals with the early aggregation of the cellular slime mold Dictyostelium discoideum [5].

When deciding how to model a system one must determine what modeling framework to use. A fundamental issue is whether to use a discrete representation, a continuous representation or some mixture of the two. This is resolved by examining the strengths and weaknesses of the different representations in the context of why the model is being developed. Continuum models are more commonly used and, perhaps as a consequence, the mathematical techniques to analyze them are readily available. Thus if the goal of the model is to understand stability, bifurcations or other general qualitative features of the system, a continuum approach will yield more insight. If on the other hand, a more quantitative model is desired with specific questions relating to local interaction, such as how the interactions of individual cells influence the system, a discrete approach may be more appropriate. Of course there are many other questions to consider. Is there a natural discrete structure to the system? Is the phenomenon being modeled a macroscopic or microscopic one? Are the interactions causing the phenomenon local or global? Are the densities such that a continuum is realistic? How is the model going to be understood analytically, through simulations or in some other way? An example of the importance of modeling approach is provided by Durrett \& Levin [7] who compare four different modeling approaches for the same biological system: a spatially homogeneous model, a reaction diffusion formulation and two models with discrete spatial structures. They find that in some circumstances the models give different results, indicating the importance of how the model is formulated. We will mention a few of the current discrete methods being used.

Discrete models can have variables that are defined on a fixed lattice, an array of discrete fixed sites connected to each other so that they have well defined neighbors, or at discrete particles which are allowed to move freely in the domain. A useful analogy can be made with two different views frequently used in fluid dynamics: Eulerian, fixed spatial coordinates, and Lagrangian, coordinates which move 
with the fluid. Although in that context these views are applied to continuum models, here we apply them to discrete modeling methods.

The first viewpoint, Eulerian, gives rise to most of the current discrete modeling, in which a fixed lattice is defined. These lattice based models include the process mentioned above of numerically solving continuum models by discretizing the domain and solving the equations based on finite differences. Here the discrete structure is imposed for numerical reasons and is not motivated by the physical system, which was originally modeled with a continuum method. In essence, these discrete computational schemes model mathematical equations and only indirectly represent the physical system. This contrasts with another group of models where an underlying discrete physical structure motivates the lattice based modeling approach. These models, while discrete in space, use continuous time variables. Typically they give rise to a large number of coupled ordinary differential equations and are commonly used in solid state physics where the crystalline structure of the material defines a natural physical lattice. The discrete nature of the models along with nonlinear interactions can give rise to solutions which are not possible in their continuum counterparts [29]. In a newer application, this method is used to model juxtacrine signaling of epithelial cells, which form a lattice on the surface of the skin and other tissues, and communicate with nearest neighbors via membrane bound proteins $[3,19]$. Although in this group the models mix a continuous time variable with discrete space variables (the lattice), they are fundamentally different from the hybrid models which are the focus of this paper and have continuous and discrete structures in the same dimension.

Cellular automata are lattice based methods which, in addition to the discrete space variable, have discrete time and a finite set of values that the dependent variable can assume. When this method was first introduced by von Neumann and Ulam, it was designed for biological applications [30]. Since then it has been used to model a wide variety of physical and biological phenomena including the evolution of galaxies, reaction diffusion systems, phase transitions, crystal growth, fluid dynamics, and the growth of organisms [34]. The cells or lattice sites can represent atoms in a crystal or regions in space. The state of the cell or the dependent variable could represent the spin of the atom, the excitability of the system, the average velocity of the fluid or whether a biological cell exists at that space location. At each time step the state of the cell can alter depending upon interactions with neighboring cells. Cellular automata have had considerable success in reproducing physical phenomena with relatively simple local rules which can be easily simulated.

The second view point which can be taken in discrete modeling is a Lagrangian view of tracing 
particles which are unconstrained in the domain. In these models, usually numerical treatments of continuum models, if a grid is defined it is defined by particle positions which change as the particles move. This gives a major disadvantage if the particles' motion with respect to its neighbors is great. The mesh deforms and particles which started as nearest neighbors are no longer close. If near neighbor interactions are important the solutions will become numerically unstable. To correct this, remeshing is employed or, more typically, hybrid numerical methods of the Lagrangian and Eulerian views are used. In the hybrid numerical method particle-in-cell, the moving or Lagrangian particles define only nodes and not a mesh, eliminating the problem. Originally the particles in this method carried mass and as they moved from one grid cell to another they transferred mass, momentum and energy to different parts of the Eulerian grid. It was on the fixed grid which defined the computational cells where the average values of velocity, pressue, energy and other quantities were calculated. The method has evolved into a group of particle-mesh methods commonly used in physics and chemistry to solve models of plasmas, fluid flow, and combustion problems $[25,11,18]$. In these methods there is both a fixed (Eulerian) grid and moving (Lagrangian) particles. The field quantities are typically solved on the fixed grid and the particle properties remain with the particles. In plasma flow this means the charges are located with the particles and the electric field is solved on the fixed grid. For combustion models the convection current is solved on the fixed grid and the reacting particles are solved using the moving particles. Here we adapt this technique and apply it to a modeling method which mixes continuum and discrete variables. We numerically solve the continuum variables on a fixed grid using finite differences and allow the discrete variables to be unconstrained in the domain.

We mention two other methods which use the Lagrangian views. One very similar to both the particle-in-cell method and our models is the immersed boundary method [20], developed for a model of the heart. This numerical method uses an Eulerian approach for the fluid flow of the blood and a Lagrangian approach for the fibers which model the heart tissue. The two main differences with our model are first, this method is a numerical one for a continuum model and second, the Lagrangian particles define a mesh (a fiber) where the connections to the original neighbors are important. The other method is given in a model developed by Weliky \& Oster [32] for epithelial morphogenesis. There the cells are represented by nodes on their boundaries. The nodes are free to move depending on the forces exerted on them. In this model the mesh defined with the nodes is important. It is essential to know which nodes belong to which cell boundary.

The remainder of the paper is outlined as follows. In the next section the general framework for the models is described after introducing the two biological applications. Then the key issue of how the 
discrete variables interact with the continuum variables is addressed. This is followed by sections 4 and 5 in which the implementational details for the wound healing and the Dictyostelium discoideum models are given. Finally we conclude with a discussion of different biological applications of this model.

\section{The Models}

The basic modeling technique used for the two models we consider in this paper is the same. In both cases it is convenient to represent some components of the systems as continuum variables and some as discrete variables. As commonly occurs when examining biological systems, there is a great disparity in space scale between interacting parts of the system, for example, herd animals with respect to their food, amoeba with respect to the chemical environment or amoeba and their substrate. While it is true that both parts of the system have a discrete structure, on the scale of the larger component, microns for the cell, the substrate can be easily and accurately modeled as a continuum, whereas the cells cannot. Of course for both our applications, we are interested in a macroscopic pattern on yet a larger scale. One could argue that at this scale the cells can also be modeled as continuum. This is the most common modeling approach, but it is the properties of individual cells acting locally that give rise to the global patterns. Thus a discrete formulation for the cells makes the models more flexible with regard to the cell properties and it is easier to explore how changing these properties alters the global results. We now introduce the two models, starting with wound healing and then Dictyostelium discoideum. After introducing the applications we describe the general mathematical framework.

\subsection{Wound Healing}

In the early 1980's it was discovered that fetal wound repair results in little or no scarring, generating significant renewed interest in the mechanisms of wound healing [1]. Although much has been learned, it is still unclear why in adults the tissue regenerates in a manner which results in scarring. The characteristic of scars on which we focus is the alignment of collagen fibers. The bulk of the dermis is composed of a fibrous protein called collagen. In normal skin this fibrous structure has a random or reticular orientation, whereas in scars it is aligned [33]. We are interested in how the fibroblasts, the cell type that produces collagen, replace the blood clot, which is composed primarily of fibrin. The obvious ingredients for the model are the fibroblasts, collagen and fibrin. Although there are many other things we could include, for simplicity we limit the model to these three components. The collagen and fibrin fibers composing the extracellular matrix are represented by a continuous vector field, but the fibroblasts 
are represented as discrete particles. The protein fibers are two to three orders of magnitude smaller than the fibroblasts and are densely spread, so that a continuum approximation is justified. We use a vector field because both the density, represented by the length of the vector, and the direction of the fibrous network are important. The wounds we consider are approximately $1 \mathrm{~mm}$ by $0.5 \mathrm{~mm}$ in size. This makes the fibroblasts one to two orders of magnitude smaller than the domain size we are considering and the densities of the fibroblasts (typically a maximum of 600 cells in the domain) are low making a continuum representation for the fibroblasts less realistic (see figure 1). In addition, when comparing the results of continuum models with the discrete formulation [17], one sees that more structure in the fiber orientation is captured when using the discrete cells.

The model focuses on the interactions of the fibroblasts with the extracellular matrix. There are four fairly simple interactions: the cell speed depends on the local protein densities, the cell direction depends on the local fiber orientation, the fiber orientation is modified by the direction of the cells and the protein densities are modified by the fibroblasts. In the model the location and direction of the fibroblasts are tracked as well as the evolution of the extracellular matrix. The matrix evolution and the cell paths are described by ordinary differential equations.

\subsection{Dictyostelium discoideum}

Dictyostelium discoideum (Dd) is a widely studied system exhibiting key features in developmental biology, including in particular the ability to move in a directed manner due to a chemical stimulus called chemotaxis. During its life cycle, the individual amoeba feeds on bacteria and multiplies by cell division. When food becomes scarce and the amoebae starve they undergo several developmental changes. Two of these changes, which occur between six and eight hours after starvation are obtaining a chemotactic sensitivity to the chemical cyclic adenosine 3',5'-monophosphate, or cAMP, and becoming relay competent. The first means they move up chemical gradients of cAMP. The second, relay competence, means that a cell can relay a signal of cAMP. In other words, if a cell is stimulated with cAMP it will produce and output its own burst of cAMP, thus relaying the signal. So if relay competent cells are spread on a substrate, they can form an excitable medium which is capable of supporting traveling waves of cAMP. If in addition the cells are chemotactically sensitive, they will reorganize themselves. Thus, due to the presence of cells which periodically emit signals of cAMP called pacemakers, the other cells aggregate towards the pacemaker and form a multicellular organism.

In this different biological setting where cell motion is key to the development of the organism, we 


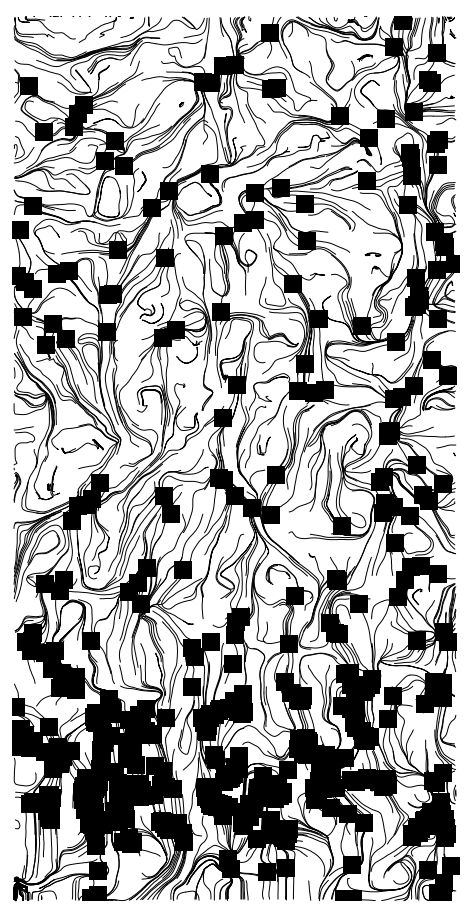

Figure 1: The collagen alignment and cell positions are shown for a typical wound healing simulation. It is clear that the density for the upper region of the wound is low and not well approximated by a continuum variable. The thin lines are stream lines for the vector field representing the collagen fibers, i.e., at each point the tangent of the line is in the same direction as the vector field. The black dots show the position of the cells and are scaled such that their area is the same as the support of the weight $w$. 


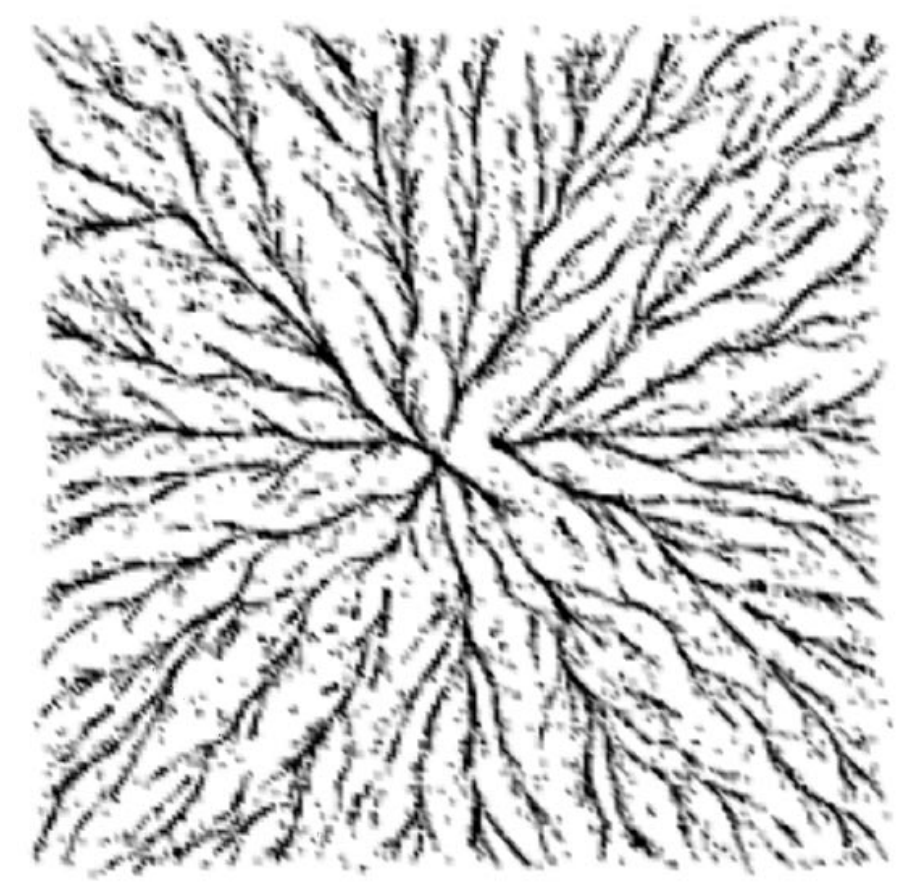

Figure 2: Aggregation patterns for a typical simulation of the Dd model. The discrete nature of the cells allows the model to easily simulate the intricate branching patterns seen during aggregation.

formulate a model of the early aggregation phase with the same basic framework used for wound healing. We represent the chemical concentration of cAMP as a continuum variable and the individual amoebae as discrete entities. Typical aggregation territories have from 10,000 to 120,000 cells in the region. Thus a continuous model would be feasible [13], but with the discrete depiction the properties of the cells are more easily modeled and altered giving the method more flexibility. This is an important feature since a major goal of the work is to understand how cell properties alter the overall density patterns. In addition the discrete representation of the cells allows the model to easily capture the detailed streaming behavior of the system (see figure 2) and the rough profile of the global cAMP wave which is the result of the relay response of each individual cell (see figure 3). The chemotactic ability of the cells is simulated using various motion rules such as: if the time derivative of cAMP exceeds a threshold the cells move, they move at a fixed speed, for a fixed time and in the direction of the gradient of cAMP. Other possible rules are described and used in [5]. The relay competence is modeled with ordinary differential equations which represent the various internal chemical complexes which are involved in the signal transduction pathway for cAMP relay. The external concentration is determined by a parabolic partial differential equation. Thus we again have individual cells which modify the continuum variable, which in turn modifies the behavior of the cells. 


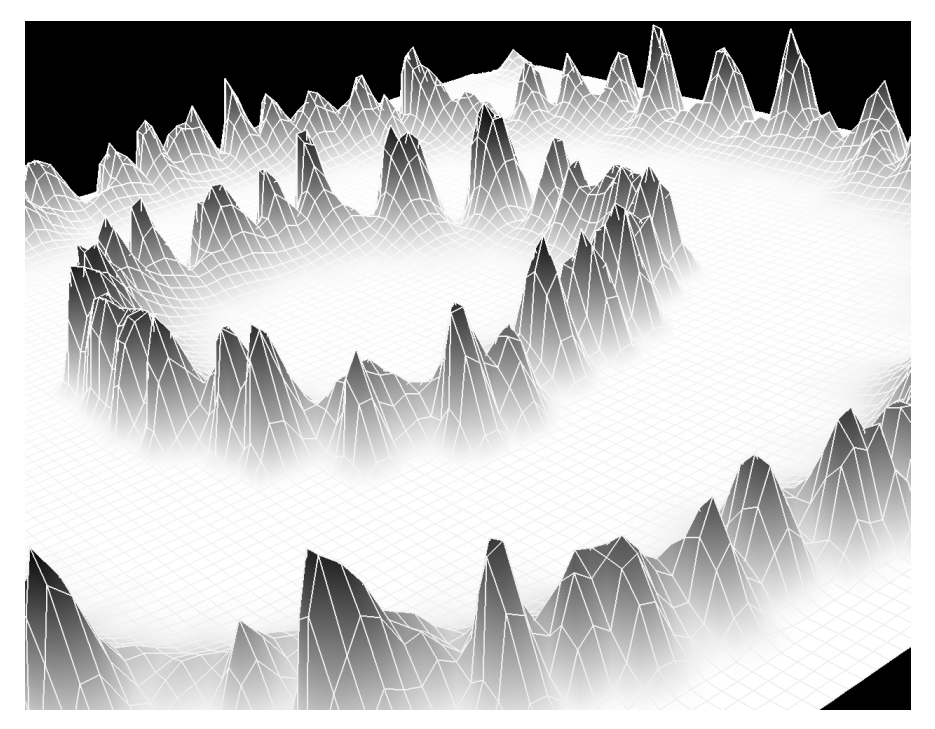

Figure 3: Here the concentration of cAMP is plotted. The rough profile is due to the high and low amoeba density regions. The discrete cell representation enhances this feature of the system.

\subsection{The General Model}

Both models fit the following general framework. Let $\mathbf{u}$ represent the continuum variable which is defined over the entire spatial domain. For wound healing, it represents the fibrous proteins of the extracellular matrix and for Dd it is a scalar and represents the concentration of cAMP. The discrete variables $\mathbf{v}^{i}$ are not defined on the spatial domain and represent properties of the cells, with the superscript $i$ denoting which cell. For example $\mathbf{v}^{i}$ could have two components representing the location of the cell and thus its range would be a subset of the spatial domain for $\mathbf{u}$. In the wound healing application $\mathbf{v}^{i}$ is a vector in $\Re^{4}$, two components representing the fibroblast location and two representing its direction. In the Dd model, $\mathbf{v}^{i}$ has ten components, two again give the cell location, one gives movement information, and the other seven represent internal chemicals which depict the state of the cell. The manner in which these variables interact with each other is determined by the system being modeled. In wound healing the system consists of coupled ordinary differential equations, while for Dd the system is made up of coupled ordinary differential equations and a partial differential equation. In general, the interaction can be represented:

$$
\begin{aligned}
\mathcal{L}_{1}(\mathbf{u}) & =\mathbf{F}(\mathbf{u})+\sum_{i=1}^{N} w\left(\mathbf{x}, \mathbf{v}^{i}\right) \mathbf{F}_{i}\left(\mathbf{u}, \mathbf{v}^{i}\right) \\
\mathcal{L}_{2}\left(\mathbf{v}^{i}\right) & =\mathbf{G}_{i}\left(\mathbf{u}, \mathbf{v}^{i}\right)
\end{aligned}
$$

where $\mathbf{x}$ is the spatial variable i.e., in $\Re^{2}$. We have in mind that $\mathcal{L}_{i}$ are linear differential operators such as differentiation with respect to time. The $\mathbf{F}_{i}$ 's describe how the cells modify the continuum variables 
and the weights $w$ determine if the cell influence is local or not. For Dd the influence is modeled as a Dirac delta function making it a point source or sink. In wound healing the weight is a localized pyramid-like function. Choosing two very different type of interpolant for similarly sized cells is due primarily to how the cells modify their environment and also to the domain size being considered. In Dd the cells modify their surroundings by degrading or outputting a diffusible substance. In wound healing the fibroblast and extracellular matrix interactions we consider are not diffusible. In addition the size scale of interest for wound healing is at least one order of magnitude smaller than that in Dd.

The numerical algorithm used to solve the system is similar to the particle-in-cell method mentioned earlier. For equation 1 the domain is discretized and values for $\mathbf{u}$ are solved on a fixed or Eulerian grid. Equation 2 is solved by treating $\mathbf{v}^{i}$ as Lagrangian particles which are unconstrained in their movement. Immediately the key issue is apparent - how can the variables interact when one, $\mathbf{u}$, is constrained to a lattice and the other, $\mathbf{v}^{i}$, is not. The solution is to interpolate the information from the lattice to the cell locations and back.

\section{Interpolators}

By letting $\overline{\mathbf{u}}$ be the discrete version of $\mathbf{u}$ and writing the difference operator approximating $\mathcal{L}_{1}$ as $\overline{\mathbf{L}}_{1}$, equations 1 and 2 become

$$
\begin{aligned}
\overline{\mathbf{L}}_{1}(\overline{\mathbf{u}}) & =\mathbf{F}(\overline{\mathbf{u}})+\sum_{i=1}^{N} \mathbf{I}\left[w\left(\mathbf{x}, \mathbf{v}^{i}\right) \mathbf{F}_{i}\left(\mathbf{T}\left(\overline{\mathbf{u}}, \mathbf{x}^{i}\right), \mathbf{v}^{i}\right)\right] \\
\mathbf{L}_{2}\left(\mathbf{v}^{i}\right) & =\mathbf{G}_{i}\left(\mathbf{T}\left(\overline{\mathbf{u}}, \mathbf{x}^{i}\right), \mathbf{v}^{i}\right),
\end{aligned}
$$

where $\mathbf{T}$ interpolates the value of $\overline{\mathbf{u}}$ to the location of the cell, $\mathbf{x}^{i}=\left(x^{i}, y^{i}\right)$ and $\mathbf{I}$ interpolates the way the cell modifies $\mathbf{u}$ to the lattice. The interpolators which are chosen will depend on the system being modeled.

The interpolators $\mathbf{I}$ and $\mathbf{T}$ are chosen to be the same in some particle-mesh simulations in physics. For problems with charged particles it can be shown that in order to conserve momentum the two interpolators must be the same. This ensures that a particle does not exert any force on itself [12].

It is worth mentioning that in multigrid methods the same problem of interpolating back and forth from different grids exists. There it is sometimes convenient to choose $\mathbf{I}$ to be the adjoint of $\mathbf{T}$, but as in our method the best choice for the interpolators is problem dependent [2]. We do not do that in our examples, although we comment on the possibility of doing so in the following subsections. Unlike 
our situation, in multigrid methods the grids are typically static structures with some being subsets of others. In addition the variables being interpolated are the same, either errors or solutions, whereas in our case they can represent items which behave very differently.

\subsection{Interpolation for the wound healing model}

First let us consider the function $\mathbf{T}$ which interpolates $\overline{\mathbf{u}}$ from the Eulerian grid to the Lagrangian particles. It gives values for a function which is continuous, but for numerical purposes has been discretized, at point off the numerical lattice. This is a standard interpolation problem and depending on the lattice structure, the accuracy and the smoothness desired, an appropriate interpolator can be chosen. We choose a tensor product interpolant using quartic Lagrangian interpolation in each direction [21] defined by

$$
\mathbf{T}\left(\overline{\mathbf{u}}, \mathbf{x}^{i}\right)=\sum_{n=-2}^{2}\left(\sum_{m=-2}^{2} \overline{\mathbf{u}}_{j-m, k-n} \ell_{j-m}\left(x^{i}\right)\right) \ell_{k-n}\left(y^{i}\right)
$$

where

$$
\ell_{j}(x)=\frac{\left(x-x_{j-2}\right)\left(x-x_{j-1}\right)\left(x-x_{j+1}\right)\left(x-x_{j+2}\right)}{\left(x_{j}-x_{j-2}\right)\left(x_{j}-x_{j-1}\right)\left(x_{j}-x_{j+1}\right)\left(x_{j}-x_{j+2}\right)}
$$

In equation $5, \overline{\mathbf{u}}_{j, k}$ is the value of $\mathbf{u}$ at the grid point $\left(x_{j}, y_{k}\right)$ which is the closest grid point to $\mathbf{x}^{i}=\left(x^{i}, y^{i}\right)$,

thus $j$ and $k$ depend on $\mathbf{x}^{i}$. The error introduced by $\mathbf{T}$ is $O\left(h_{x}^{5}\right)+O\left(h_{y}^{5}\right)$ where $O(x) \propto x$ and $h_{x}$ and $h_{y}$ are the mesh sizes in the $x$ and $y$ directions respectively.

Now we consider the function $\mathbf{I}$. The situation is very different when interpolating from the Lagrangian particles to the Eulerian grid. In our models the Lagrangian particles are not the discretized version of a continuum variable, rather they are inherently discrete particles. The behavior of $\mathbf{I}\left[w \mathbf{F}_{i}\right]$ depends on the physical system being modeled and on how the discrete particles interact with the continuum variables. Thus using a standard interpolant may not make sense. In fact, since there may be one Lagrangian particle surrounded by several Eulerian grid points, there is one known value and several unknown values. Typically, interpolation problems are cases where there are several known values. A more intuitive way to handle the problem is to think how the discrete particles influence the continuum variable. This will define the weight function $w$ and help determine what type of interpolation is sensible. For example, provided that the support of $w$ is larger than the mesh size for the Eulerian grid, $\mathbf{I}\left[w \mathbf{F}_{i}\right]$ can simply be $w$ evaluated on the grid or $\mathbf{I}$ could be chosen as the adjoint of $\mathbf{T}$. In wound healing the effect of the cells on the extracellular matrix is very different from the effect of the matrix on the cells giving little justification for the latter example. By choosing $\mathbf{I}$ to be the restriction of $w$ to 
the grid, the larger the support of $w$ when compared with the mesh size the better $\mathbf{I}\left[w \mathbf{F}_{i}\right]$ approximates the behavior of $w \mathbf{F}_{i}$. If the support of $w$ is not large when compared to the mesh size, $\mathbf{I}$ needs to be considered carefully. This will be demonstrated when we treat the interpolations used for Dd.

In the wound healing example, we take $\mathbf{I}\left[w \mathbf{F}_{i}\right]$ to be $w \mathbf{F}_{i}$ defined on the lattice. Recall that the Lagrangian particles are fibroblasts. They modify the extracellular matrix primarily through processes localized at the cell surface. For the most realistic model, the support of $w$ should coincide with the shape of the fibroblasts. The next approximation would be to have an elongated support in the direction of the fibroblasts' motion. For simplicity we take the support to be a square region with sides of length 20 microns. We choose a piecewise linear function whose maximum is at the cell location defined by

$$
w\left(\mathbf{x}, \mathbf{x}^{i}\right)=a_{x} a_{y}
$$

where

$$
\begin{aligned}
& a_{x}=\max \left(1-\frac{\left|x^{i}-x\right|}{L}, 0\right) \\
& a_{y}=\max \left(1-\frac{\left|y^{i}-y\right|}{L}, 0\right) .
\end{aligned}
$$

Here $L$ is a parameter that determines the support of $w$ and is taken to be 10 microns. A graph of $w$ is shown in figure 4.

\subsection{Interpolation for the Dd model}

The interpolator from the Eulerian grid to the Lagrangian particles used in the Dd application is the scalar version of $\mathbf{T}$ defined in equations 5 and 6 .

In the Dd model $I$, the interpolator from the Lagrangian particles to the Eulerian grid, is a real valued function and not vector valued (thus we change from $\mathbf{I}$ to $I$ ). The Dirac distribution is the weight for the influence of the cells. So in this case, the support of the weight function is smaller than the mesh size. The approach taken for defining this interpolator in the wound healing model fails here because the cell would only modify the cAMP concentration when its location coincides with a grid point. Clearly that is not acceptable. Instead we choose $I$ to have properties which are consistent with the underlying model. Recall that the amoebae influence the concentration of cAMP by acting as point sources or sinks. We choose $I$ to have the following three features:

(i) $I\left[F_{i}\right]\left(x^{i}, y^{i}\right)=\frac{F_{i}}{h_{x} h_{y}}$. 


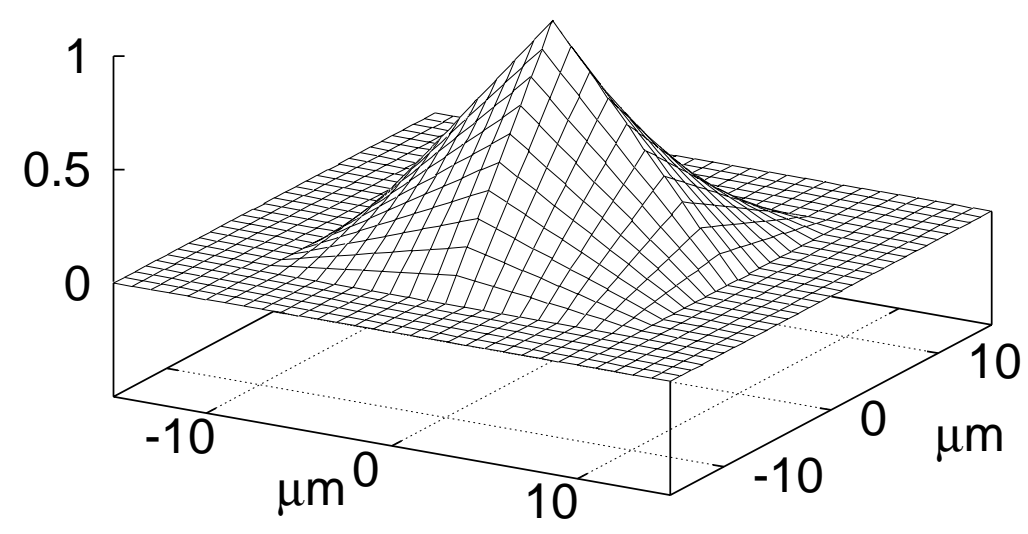

Figure 4: Graph of $w$ for the wound healing model.

(ii) $\int_{\mathrm{R}^{2}} I\left[F_{i}\right] d A=F_{i}$ (we define $I$ to be zero where it is not positive).

(iii) $I$ should decrease from $\left(x^{i}, y^{i}\right)$ at the same rate in the $x$ and $y$ directions.

By restricting $I$ to be a tensor product interpolation which is a continuous, bivariate, piecewise linear polynomial, the three conditions above uniquely define the interpolant. The second condition ensures that the amount of chemical produced (or degraded) by the cell is conserved. The other two ensure that only the four nearest grid points are in the support of $I$ and the maximum value of $I$ is attained at the cell location. The third property also implies that the cAMP diffuses at the same rate in both directions. If the amoeba is located exactly at a grid point, $I$ acts as the Dirac distribution.

The interpolant $I$ is defined as

$$
I\left[F_{i}\right](x, y)=a_{x} a_{y} F_{i}
$$

where

$$
\begin{aligned}
& a_{x}=\max \left(\frac{h_{x}-\left|x^{i}-x\right|}{h_{x}^{2}}, 0\right) \\
& a_{y}=\max \left(\frac{h_{y}-\left|y^{i}-y\right|}{h_{y}^{2}}, 0\right) .
\end{aligned}
$$

Geometrically we think of the interpolation as being proportional to the complementary area of the point to which we are interpolating (see Figure 5). This interpolant is similar to the one used in the 


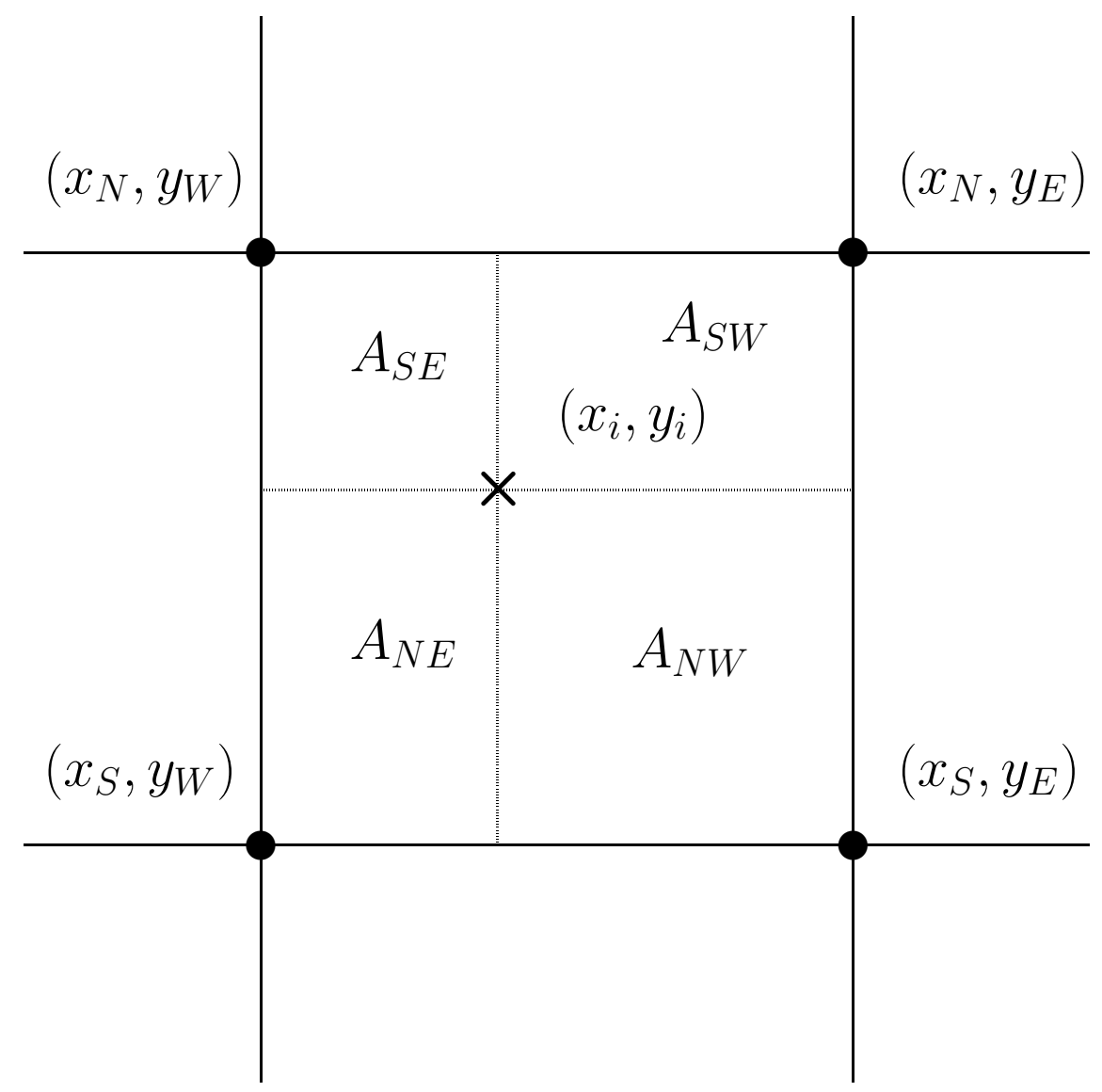

Figure 5: The complementary areas are shown for each of the four surrounding grid points. The location of the cell is marked by $\times$ and the grid points are marked by $\bullet$. $A_{S W}$ is the complementary area for the grid point $\left(x_{S}, y_{W}\right)$.

wound healing but has a fundamental difference. Here the support of $I$ depends on the mesh size. In fact, the sequence of interpolants $I_{n}[1]$ where $h_{x}=h_{y}=\frac{1}{n}$ is a delta sequence [15] meaning that

$$
\lim _{n \rightarrow \infty} \int I_{n}[1] d A=1
$$

This is exactly the property we want since we are trying to interpolate the Dirac distribution. The particle-mesh method cloud-in-cell uses the same type of interpolation [12].

This interpolation of the Dirac distribution, depending on the grid size, the time step and the diffusion coefficient, has the effect of more rapidly or more slowly diffusing the contribution of the amoebae to the grid. Consider an initial point source in the plane that satisfies the heat equation with the same diffusion coefficient used in our model. After the largest time step used in our numerical simulations, approximately ninety percent of the diffusing substance is contained in the circle centered 


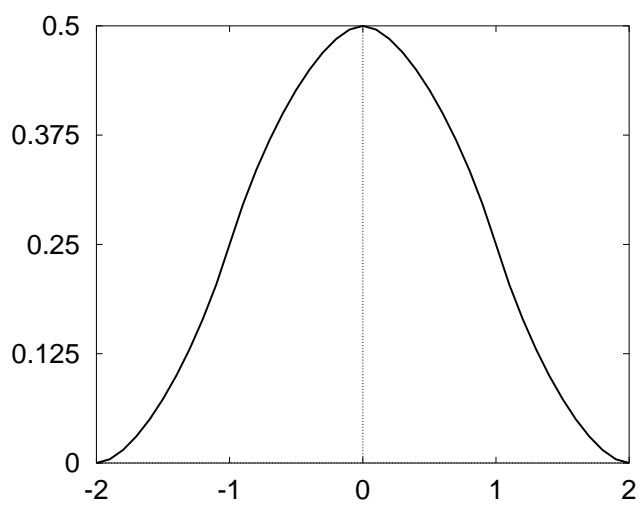

Figure 6: Graph of $\phi(r)$.

at the source with diameter equal to the mesh size. This shows that our interpolation initially speeds up the diffusion of cAMP until it reaches the grid. The error introduced by $I$ can be either considered as temporal, spatial or both in the numerical algorithm.

In this case an alternate choice of $T$ would be the adjoint of $I$. Since both $T$ and $I$ interpolate the chemical concentration of cAMP from one grid to another, using an interpolator which is symmetric and thus behaves in a similar manner makes some sense. We opted to use a more accurate interpolation when feasible (from the more dense regular Eulerian grid to the sparse Lagrangian particles).

\subsection{Immersed-Boundary Method Interpolator}

We conclude this section with another interpolant developed by Peskin \& McQueen [20]. Although we do not use this interpolant, it further illustrates how the problem dictates the type of interpolant which should be used. This interpolant also approximates the Dirac function and was developed to satisfy certain properties determined by the physical system being modeled - blood flow in the heart. The authors defined a smoothed $\delta$-function in three dimensions as

$$
\delta_{h}(\mathbf{x})=h^{-3} \phi\left(\frac{x_{1}}{h}\right) \phi\left(\frac{x_{2}}{h}\right) \phi\left(\frac{x_{3}}{h}\right)
$$

where $\mathbf{x}=\left(x_{1}, x_{2}, x_{3}\right)$. A graph of $\phi$ is shown in figure 6 . The function $\phi$ is uniquely defined by five properties of the interpolant including small support, conservation of momentum and angular momentum and ensuring that the force exerted by one fiber point on another is no greater than the force on itself. 


\section{Numerical and Implementational Details for the Wound Healing Model}

In the model for wound healing, equation 1 is

$$
\begin{aligned}
\frac{d \angle \mathbf{c}}{d t} & =\kappa\|\mathbf{f}\| \sin (\angle \mathbf{f}-\angle \mathbf{c}) \\
\frac{d\|\mathbf{c}\|}{d t} & =\left(p_{c}-d_{c}\|\mathbf{c}\|\right) \sum_{i=1}^{N} w\left(\mathbf{x}, \mathbf{f}^{i}\right) \\
\frac{d\|\mathbf{b}\|}{d t} & =-d_{f}\|\mathbf{b}\| \sum_{i=1}^{N} w\left(\mathbf{x}, \mathbf{f}^{i}\right)
\end{aligned}
$$

where

$$
\mathbf{f}(\mathbf{x}, t)=\sum_{i=0}^{N} w\left(\mathbf{x}, \mathbf{f}^{i}(t)\right) \frac{\mathbf{f}^{i}(t)^{\prime}}{\left\|\mathbf{f}^{i}(t)^{\prime}\right\|} .
$$

Here $\mathbf{c}$ represent the collagen fibers, $\mathbf{b}$ represents the fibrin fibers (the blood clot) and these two vectors taken together form $\mathbf{u}$ (in equation 1), whereas $\mathbf{f}^{i}$ represents the path of the fibroblasts and thus forms part of $\mathbf{v}^{i}$ (in equation 2). The parameters $\kappa, p_{c}, d_{c}$ and $d_{f}$ are positive constants, the prime denotes differentiation with respect to time and $\angle \mathbf{c}$ denotes the angle of the vector $\mathbf{c}$. Equation 2 is given by

$$
\begin{aligned}
\mathbf{f}^{i}(t)^{\prime} & =s\left(\left\|\mathbf{c}\left(\mathbf{f}^{i}(t)\right)\right\|,\left\|\mathbf{b}\left(\mathbf{f}^{i}(t)\right)\right\|\right) \frac{\mathbf{w}^{i}(t)}{\left\|\mathbf{w}^{i}(t)\right\|} \\
\mathbf{w}^{i}(t) & =(1-\alpha) \mathbf{c}\left(\mathbf{f}^{i}(t)\right)+\alpha \mathbf{b}\left(\mathbf{f}^{i}(t)\right),
\end{aligned}
$$

where $\alpha$ is a positive constant and the function $s$ is the speed of the cells which depends on the density of the fibrin and collagen at the cells location. The numerical algorithm used to solve these equations is as follows:

(i) Interpolate (using $\mathbf{T}$ ) the magnitude of the collagen and fibrin as well as the direction of the collagen to the fibroblast locations.

(ii) Interpolate (using $\mathbf{I}$ ) the infuence of the fibroblasts to the extracellular matrix (equation 16).

(iii) Determine the fibroblasts direction (equation 17) using an explicit Euler method.

(iv) Solve the direction and magnitude of the collagen and the magnitude of the fibrin (equations 15) on a fixed grid using an explicit Euler method.

(v) Move the fibroblasts to their new locations. 


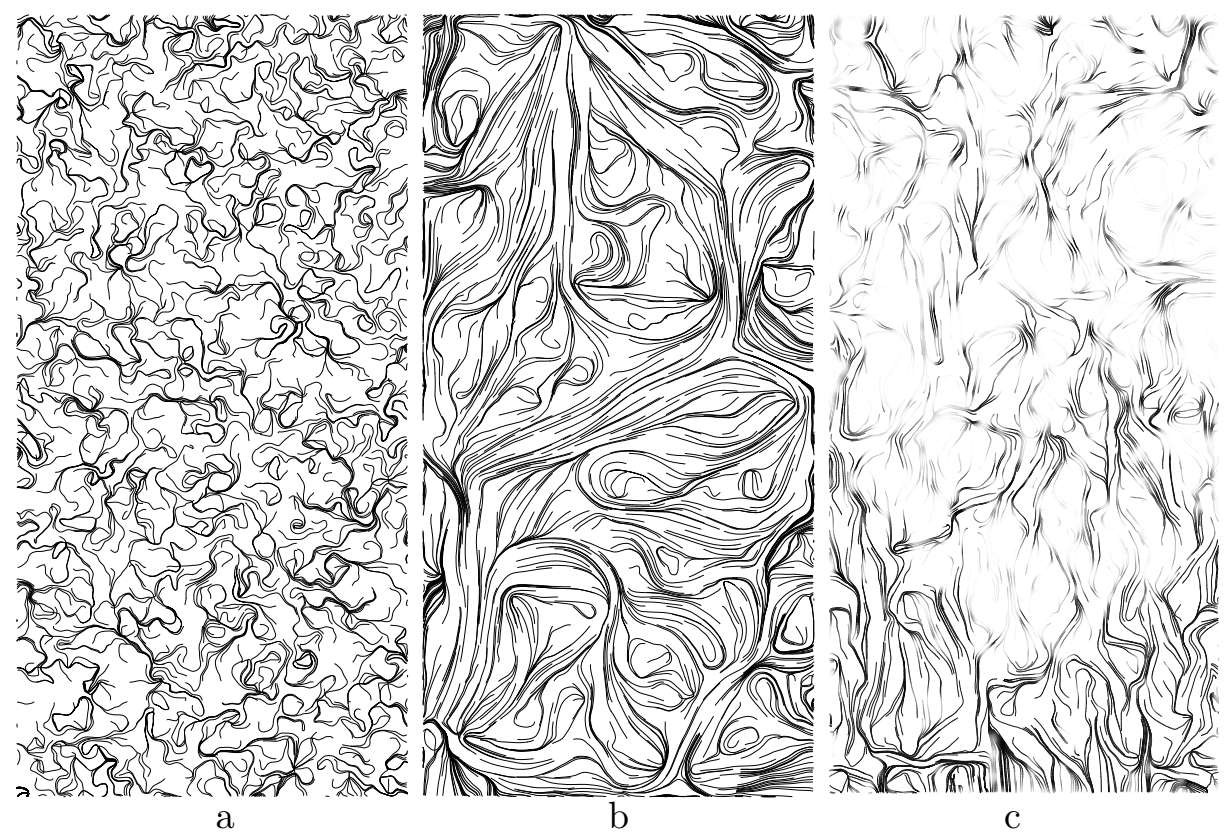

Figure 7: The collagen orientations for typical simulations. In (a) the initial random collagen orientation is shown and in (b) the collagen orientation is shown after 100 hours of remodeling by the fibroblasts on a domain of $0.5 \mathrm{~mm}$ by $1.0 \mathrm{~mm}$. In (c) the collagen alignment and density are shown in a wound region with the right and left boundaries representing interfaces with normal dermis, the bottom representing the interface with the subcutaneous fascia and the top representing the interface with the epidermis. The alignment is shown by drawing lines whose tangent corresponds to the collagen vector field (the streamlines) with black representing high collagen density and white representing low collagen density.

\subsection{Wound Healing Model Results}

In figure 7 we show typical results of the wound healing model. Figure 7(a) shows an example of a random initial orientation for either the collagen or the fibrin fibrous networks. In 7(b) a simulation is shown where there is no fibrin, thus the cells simply remodel and give more structure to the collagen matrix. After 100 hours the fibroblasts have considerably altered the original collagen configuration. The degree to which they smooth the vector field depends on several factors. Figure $7(\mathrm{c})$ shows a simulation of wound healing where the blood clot composed of fibrin is being replaced with collagen in the process of tissue regeneration. The initial conditions are given by a randomly oriented fibrin clot with fibroblasts entering from the periphery. 


\subsection{Numerical Verification}

A standard technique to test numerical solutions is to compare results using different time and/or space steps. Usually the numerical analysis predicts that the solutions will differ by an error in a manner which depends on the ratio of the different time and space steps. In this application reductions in the space step will behave in the typical manner with the error between the solutions getting smaller as the space step is decreased, but due to the discrete cells and the way they move, reductions in time step are more difficult to compare. When the time step is changed the fibroblasts sample the extracellular matrix at different points, move in different directions along different paths and alter different regions of the extracellular matrix. This means that two solutions which differ only by the time step used can be entirely different. But one still expects that the solution should converge to a limit as the time step goes to zero, although this limit may be a less biologically relevant solution than one with a finite time step. Let $\overline{\mathbf{c}}_{k}$ be the numerical solution of equations 15 and 17 where the time step is $k$. We define the following error function:

$$
\operatorname{error}(\mathbf{x})= \begin{cases}1, & \left|\angle \overline{\mathbf{c}}_{k 1}-\angle \overline{\mathbf{c}}_{k 2}\right| \geq 0.1 \\ 0, & \text { otherwise. }\end{cases}
$$

A comparison of the support of error as the time step decreases shows that indeed the solution is converging to a limit. Figure 8 shows such a comparison and one can see that the support of the error function gets smaller as the time steps decrease. Equally instructive is a comparison of the individual fibroblast paths as the time step is changed. In figure 9 one can see that some tracks converge as the time step decreases while others diverge. This is primarily due to the random nature of the initial vector field representing the extracellular matrix. It is random on a scale of 80 microns, and since the cell location are graphed every 40 microns, differences are easily seen. Still on average the paths converge and if the initial vector field is random on a coarser scale (every 160 microns), the paths are even more similar and converge quickly as expected (see figure 10).

\subsection{Cell Division}

One other implementational detail which is due to the discrete nature of the cells involves cell division. Since the model is deterministic, when two cells resulting from cell division are at the same location, they will behave identically. One way to correct this is by separating the cells. We randomly place the new cell on a circle of radius 10 microns centered at the old cell. It is obvious that the separation of the cells is important in determining how this feature affects the overall model results. The cell paths will 


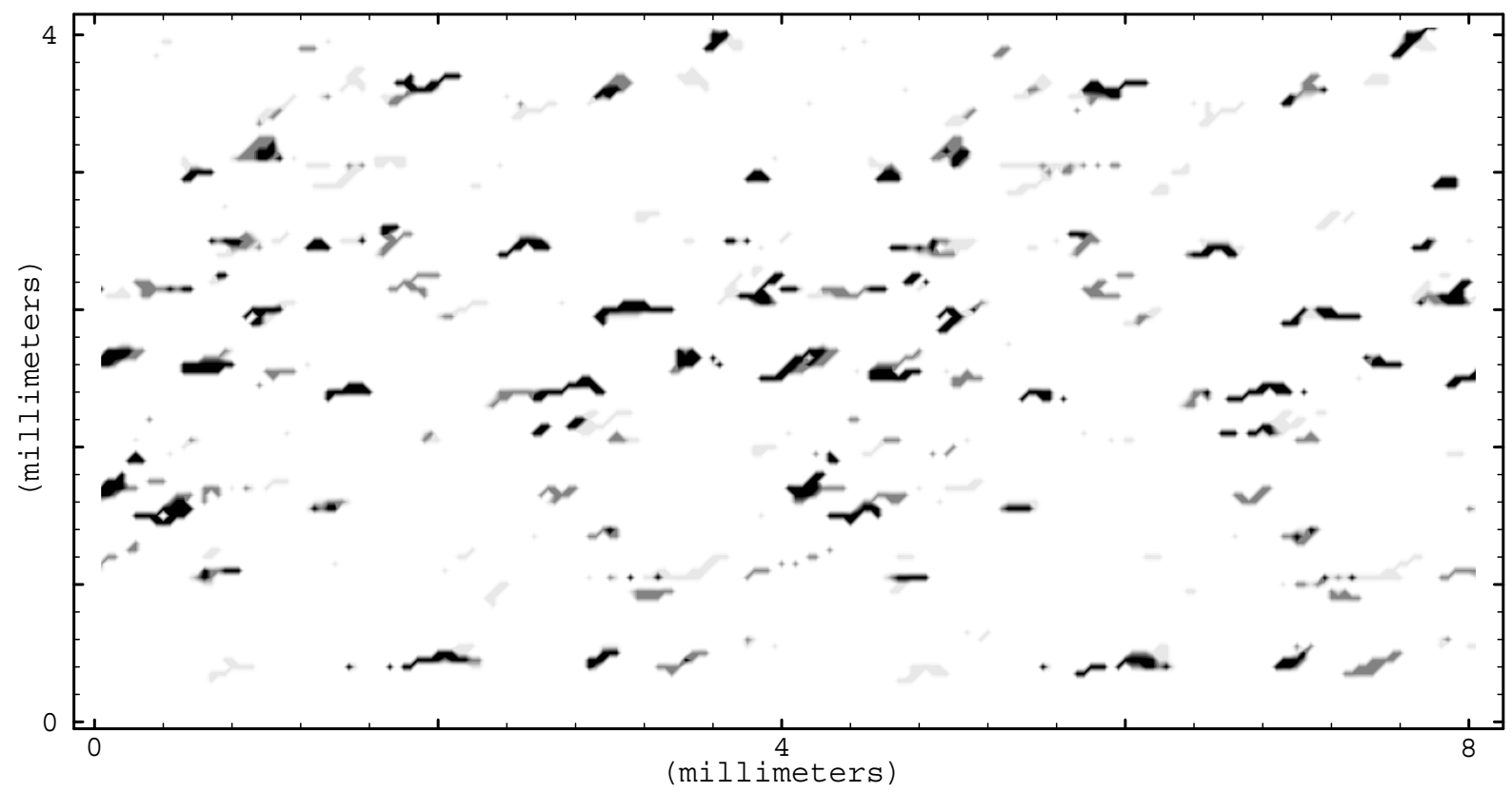

Figure 8: The shaded regions denote the support for the function error. The black corresponds to error with $k 1=.005$ and $k 2=.0025$, the medium grey corresponds to $k 1=.01$ and $k 2=.005$ and the light grey to $k 1=.02$ and $k 2=.01$. The support of error is decreasing as the time step gets smaller. The simulations were run to 10 hours with cell speeds fixed at 40 microns per hour. One hundred cells are uniformly placed in the domain of $8 \mathrm{~mm}$ by $4 \mathrm{~mm}$. 

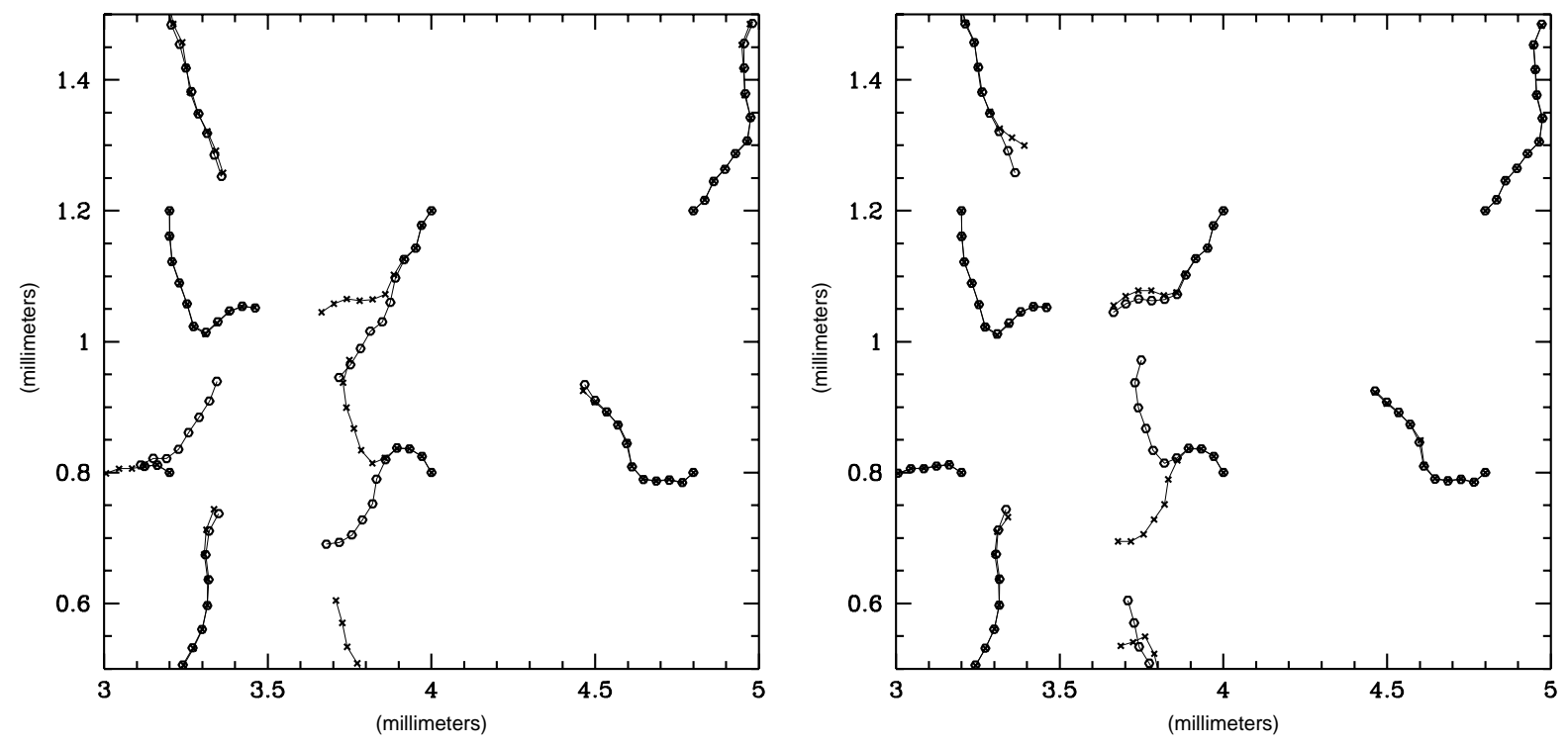

a

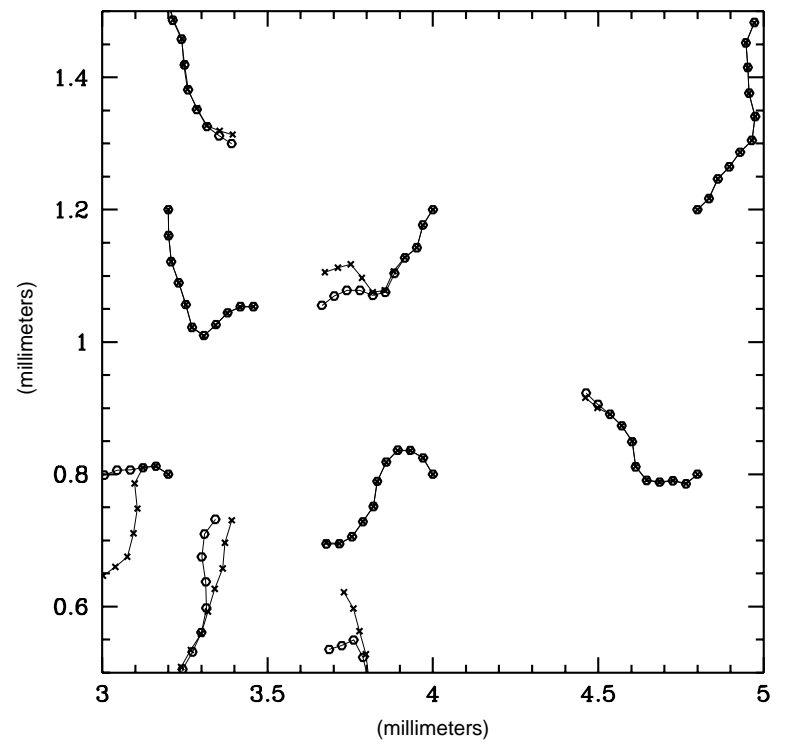

$\mathrm{C}$

Figure 9: The cell tracks are shown for simulations using different time steps. Although some paths diverge as the time step becomes smaller, overall the paths are converging. In (a) the time steps represented are .0025 and .005, in (b) .005 and .01 and in (c) .01 and .02. The symbol (x) denotes the position of the cell with the larger time step and (o) denotes the position of the cell with the smaller time step. The cell tracks remain similar until the gradual separation and the random initial conditions drastically alter the cell courses. The simulations were run to 10 hours with the cell speed fixed at 40 microns per hour and the position graphed every hour. The initial vector field, identical for all three simulations, is random on a scale of 80 microns. The axes are scaled in millimeters. 

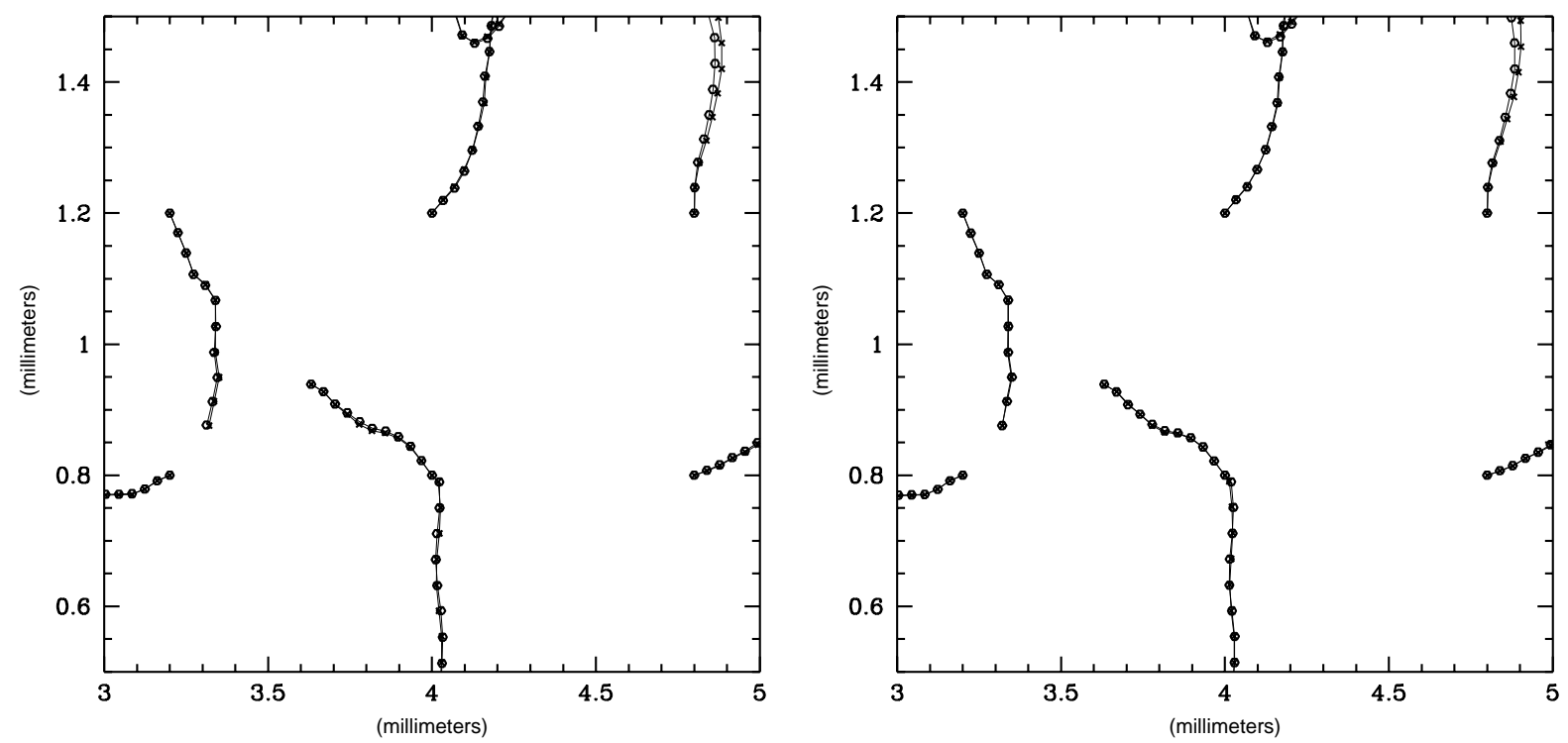

$\mathrm{a}$

b

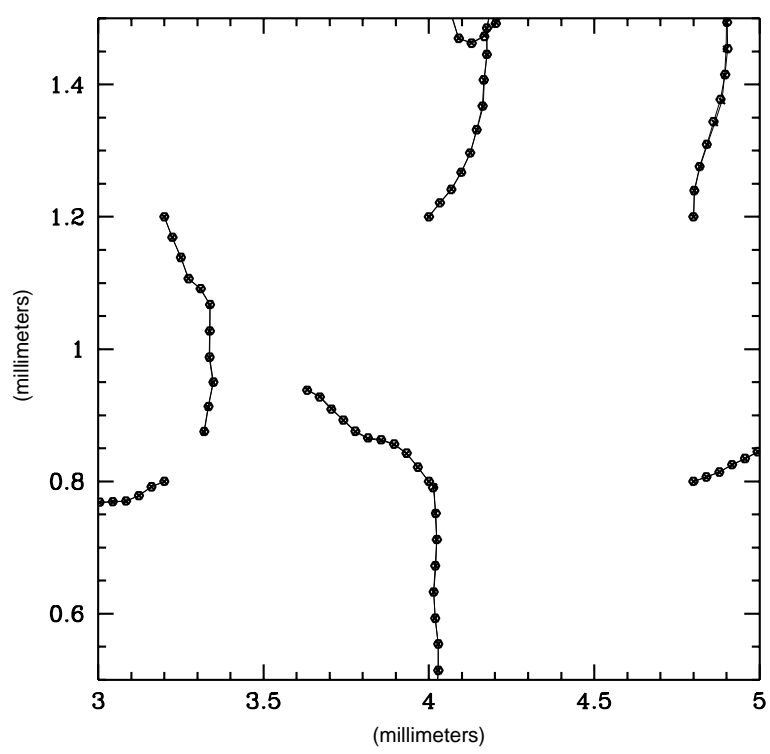

$\mathrm{C}$

Figure 10: The cell tracks are shown for simulations using different time steps, with smoother initial conditions than used in Figure 9. The cell paths all converge as the time step decreases. In (a) the time steps represented are .02 and .01 , in (b) .01 and .005 and in (c) .005 and .0025. The (x) denotes the position of the cell with the larger time step and the (o) denotes the position of the cell with the smaller time step. The simulations were run to 10 hours with the cell speed fixed at 40 microns per hour and the cell position graphed every hour. The initial vector field, identical for all three simulations, is random on a scale of 160 microns. The axes are scaled in millimeters. 
be closer to each other if the radius chosen is small when compared to the scale at which the collagen field is random. Also the simulation will behave differently if the cells are placed within the support of the weight function $w$ than if they are not. In practice, when cells divide they round up and we assume that they lose the internal structures which predisposed them to continue in the direction they where moving. In the model, the cell direction changes from the current direction according to equation 17. Thus we simulate the biology by giving each cell a new direction determined by the extracellular matrix. There are other options we could have used including randomly altering the cells' directions in some manner causing their paths to diverge.

\section{$5 \quad$ Numerical and Implementational Details for the Dd Model}

The specific form of equation 1 for the Dd model is given by

$$
\begin{aligned}
\frac{\partial u(\mathbf{x})}{\partial \tau}= & D \nabla^{2} u(\mathbf{x})-\gamma_{9} \frac{u(\mathbf{x})}{u(\mathbf{x})+\gamma_{8}} \\
& +\sum_{i=1}^{N} \frac{V_{c}}{V_{o}} \delta\left(\mathbf{x}-\mathbf{x}^{i}\right)\left(s r\left(v_{7}^{i}\right)-\gamma_{7} \frac{u(\mathbf{x})}{u(\mathbf{x})+\gamma_{6}}\right)
\end{aligned}
$$

where $u$ is the cAMP concentration, $\nabla^{2}$ is the Laplace operator in two dimensions, $s r$ is a non decreasing function, $D, \gamma_{6}, \gamma_{7}, \gamma_{8}, \gamma_{9}, V_{c}$, and $V_{o}$ are positive parameters. Equation 2 takes the following form:

$$
\begin{aligned}
v_{1}^{i} & =\frac{\alpha_{0} u\left(\mathbf{x}^{i}\right)+\left(\beta_{5}-\alpha_{0} u\left(\mathbf{x}^{i}\right)\right) v_{6}^{i}}{\alpha_{1}+\alpha_{0} w_{5}\left(\mathbf{x}^{i}\right)+\beta_{4} v_{5}^{i}} \\
v_{2}^{i} & =\frac{\alpha_{2} \alpha_{3} c_{1} v_{1}^{i}\left(1-v_{4}^{i}\right)}{1+\alpha_{4}+\alpha_{2} \alpha_{3} c_{1} v_{1}^{i}-\alpha_{4} v_{4}^{i}} \\
v_{3}^{i} & =\frac{\beta_{0} w_{5}\left(\mathbf{x}^{i}\right)}{\beta_{1}+\beta_{0} w_{5}\left(\mathbf{x}^{i}\right)} \\
\frac{d v_{4}^{i}}{d \tau} & =\alpha_{4} v_{2}^{i}-v_{4}^{i}-\alpha_{4} v_{2}^{i} v_{4}^{i} \\
\frac{d v_{5}^{i}}{d \tau} & =\beta_{2} \beta_{3} c_{2} v_{3}^{i}-\beta_{5} v_{5}^{i}+\beta_{6} c_{3} v_{6}^{i}-c_{3} \beta_{4} v_{1}^{i} v_{5}^{i}-\beta_{2} \beta_{3} c_{2} v_{3}^{i}\left(v_{5}^{i}+c_{3} v_{6}^{i}\right) \\
\frac{d v_{6}^{i}}{d \tau} & =-\left(\beta_{5}+\beta_{6}\right) v_{6}^{i}+\beta_{4} v_{1}^{i} v_{5}^{i} \\
\frac{d v_{7}^{i}}{d \tau} & =\gamma_{1} \gamma_{2} v_{4}^{i}+\Gamma_{5}\left(1-\Gamma_{7} v_{4}^{i}\right)-\gamma_{4} \frac{v_{7}^{i}}{v_{7}^{i}+\gamma_{3}}-\operatorname{sr}\left(v_{7}^{i}\right) .
\end{aligned}
$$

The $\alpha_{i}{ }^{\prime}$ s, $\beta_{i}$ 's, $\gamma_{i}$ 's, $\Gamma_{i}$ 's and $c_{i}$ 's are constant positive parameters and are defined in Dallon \& Othmer [5]. The algorithm we have developed to solve these equations can be summarized as follows. Given the initial cell states and the cell distribution, which may be uniform or random, in a square domain, with a particular initial distribution of extracellular cAMP, we perform the following steps. 
(i) Solve the extracellular equation (19) on a regular grid, using an Alternating-Direction Implicit (ADI) method for the partial differential equation, lagging the secretion term.

(ii) Interpolate cAMP from the grid to the cell positions (using $\mathbf{T}$ ) and update the intracellular variables (20) by an implicit scheme.

(iii) Update cell movement. If a cell is not moving, should it begin to move? If so, compute the direction and start movement. If it is moving, should it continue?

(iv) Transfer the secreted cAMP to the grid (using $I$ ) and repeat the cycle.

\subsection{Dd Simulation Results}

The two variables we are most interested in are the extracellular cAMP and the cell density. The cell density is the biologically visible variable which forms the patterns of interest. In figure 11 we show the cell density after 150 minutes of simulation. The cells have formed streams and are aggregating into the two circular cores. This is caused by two spiral waves of cAMP which cells are producing due to their excitability. The cAMP waves are also of interest and help explain the features of the

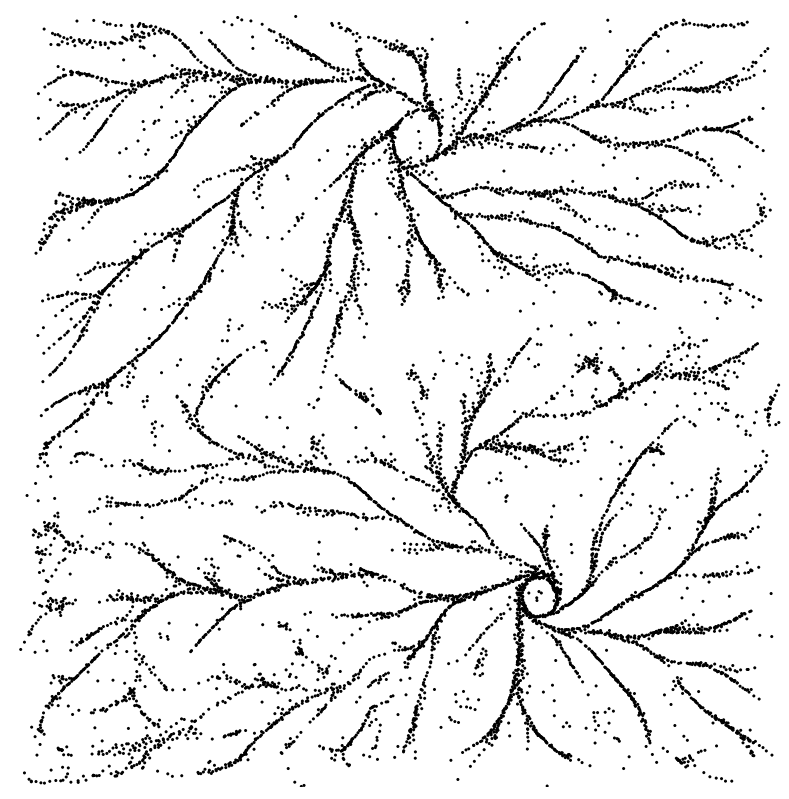

Figure 11: Density plot for a simulation at time 150 minutes. Initially 10089 cells where randomly placed on the domain. Two spiral waves form and cause the cell to aggregate and form two circular cores. The parameters are such that approximately $0.1 \%$ of the cells are pacemakers and they are randomly located. These computations use a 101 by 101 grid. 
cellular aggregation. Typically the cAMP waves are generated at pacemaking cells and expand outward as one would expect in an excitable media. They can be very rough depending on the underlying cell density (see figure 3). Thus a typical simulation starts out with a roughly uniform density of cells. The oscillatory cells initiate a cAMP wave which propagates outward into the excitable media formed by the cells. The cells then start to move and organize themselves according to the cAMP wave. As they move and aggregate they change the excitability of the system by changing the cell density. Thus the low density regions eventually do not propagate cAMP waves.

\subsection{Verification of the Numerical Scheme}

The standard method for showing convergence of a numerical scheme for a linear problem is to prove that it is consistent and stable, which is equivalent to convergence by the Lax equivalence theorem [22]. This is the approach we have taken for the linear portion of the model without the summation term in equation 19. Consistency is easily seen by expanding the discretized equations about a point using Taylor series and proving that the local truncation error approaches zero as the mesh size approaches zero. The stability of the scheme can be proved using von Neumann's method where the initial conditions are expressed in a finite Fourier sum and then determining if the terms are amplified [26]. We can further show, using results from Hundsdorfer \& Verwer [14], that the scheme including the nonlinear term in equation 19, but still without the summation, is unconditionally stable and convergent. Due to the discrete model formulation, the Dirac delta distribution is included as a weight in the summation and causes difficulties in performing any standard local truncation error analysis. Some progress can be made by locally integrating the error and thus defining a somewhat paradoxical "averaged" local truncation error. This approach does not distinguish between different interpolants as one would hope, provided that they have the limiting property of a delta sequence (see equation 13). It is likely that for the purposes of numerical analysis and the type of limiting argument used, the discretized equation will be consistent with almost any interpolator which approximates the Dirac distribution. In order to determine which type of interpolation is most appropriate, the physical characteristics of the system being modeled must be considered.

Once the model is formulated and the numerical scheme has been developed, an important issue is testing it. In this case, there are several ways to test different parts of the algorithm. The movement rules and internal variables $\mathbf{v}^{i}$ are easily tested with various functions which specify $u$. In this way it is easy to determine that the cells are moving in the appropriate directions and at the right times. 
Likewise, the ADI scheme and the interpolators are tested on known problems. In order to test the combined effect of several components in the model, the cells are fixed at the grid sites. This makes the model equivalent to a continuum description developed by Tang \& Othmer [27] and the results can be compared. The waves of cAMP which are generated have the same amplitudes, speeds and periods for both models and compare nicely to experimental results. The full model is tested by using two different order discretizations of the Laplace operator. In these tests, a comparison of the results shows significant differences in the solutions with the lower order scheme showing more grid effects than the higher order scheme. This indicates that errors from the interpolators are not dominating the simulation results. The final test is to determine if the results of the entire model are consistent with the biological system, which they are.

\subsection{Cell Adhesion}

We include a feature of the model which is allowed by the discrete representation of the cells. In order to simulate the biology more closely and to save computation time, the cells are combined when then are within a radius of 5 microns of one another, and then they are treated as one cell with twice the strength. For example if cell $j$ and $k$ are are close enough to adhere, then in equation 19 the $j$ th term of the summation is deleted and the $k$ th term is multiplied by two. This is biologically reasonable since cells tend to adhere to one another when they come in contact [24]. The contact radius is chosen in an arbitrary manner erring on the conservative side. In reality, Dd amoebae become quite elongated, making a contact distance difficult to determine. The major problem with this feature is that as cells adhere they become artificially compact. The effects of this will be increased as the simulation runs for longer periods of time, so that the local densities increase causing more adhesion. Numerical tests indicate that combining cells has only a minor impact on the simulations in the time frame we are considering (150 minutes).

\section{Discussion}

In this paper we have described a modeling method which mixes continuum variables with discrete variables. To numerically solve the model an algorithm similar to the particle-in-cell methods is used where the interactions between the two types of variables are interpolated back and forth. This type of formulation tries to capitalize on the benefits from each type of representation. The continuum representation is usually numerically less intensive to calculate and can accurately describe high density 
interactions in a qualitative manner. In addition there is a vast mathematical theory developed that allows the continuum formulation to be analyzed and understood. The discrete structure may model the fundamental interactions of the system more accurately and allow more flexibility in understanding how these interactions affect the system as a whole.

The benefits and limitations of this hybrid type of modeling can be clearly seen by comparing models of the same system which use the different formulations. In Dallon \& Othmer [5] and Hofer \& Maini [13], the early aggregation of Dd is modeled using reaction diffusion equations. In the first model (described in Section 2) the hybrid approach is used, whereas in the second a continuum approach is used. Both models give insight into how the global density patterns are formed; the hybrid model is closely tied to experimental results and provides insight into the mechanisms that the cells use to move; the continuum model sheds light on the formation of the linear instabilities in the system and the characteristic spatial scales observed. In fact, each model addresses questions that, due to the different formulation, the other model cannot answer. Similarly in Olsen et. al. [17] the usefulness of the hybrid approach for wound healing is demonstrated. There the orientation of collagen in wound healing is modeled using a continuum description with a brief comparison of results obtained using the hybrid model discussed here. In this work one can see how the different model formulations highlight different features of the system. The discrete approach is able to capture collagen structures on small spatial scales which are observed biologically. The continuum models represent averages and cannot reproduce the detailed structures. Durrett \& Levin [7] also showed how different features of a system are captured by the model formation. They model a predator prey system using first continuum then discrete spatial structures. By using discrete structures, as in the hybrid method presented in this paper, questions which continuum models cannot address are answered.

Although discrete formulations have been widely applied in the physical sciences, there are many biological applications which are well suited to a discrete approach. In particular the hybrid method described here can be used in many areas where cell motion is important. Models involving taxis are good examples, including chemotaxis as in the Dd application, haptotaxis as in the wound healing application and Boyden chamber experiments. Other systems which could easily be modeled in this manner are cancer growth, tumor invasion and angiogenesis. This model formulation can also be applied to ecological modeling where larger organisms including animals can be represented naturally as discrete structures. For instance, in the work of Lewis et. al. [16] wolves and scent markings are modeled using a continuum formulation to learn about territories. This system would fit nicely into the hybrid method described here with the wolves modeled as discrete entities and the scent markings 
modeled as continuum variables.

Depending on the level one wishes to examine, models can range from a totally discrete representation, as in cellular automata, to a continuum formulation. Here we have described a method which combines the two formulations focusing on the discrete structure of some components and using a continuum representation for others.

Acknowledgements. The author would like to thank Dr. Philip Maini, Professor Jonathan Sherratt and Professor Hans Othmer for their help in developing the models discussed. In addition, I would like to thank J. Sherratt for his insightful suggestions. This work was supported by grant GR/K71394 from the ESPRC.

\section{References}

[1] Adzick, N. S., And Longaker, M. T., Eds. Fetal Wound Healing. Elsevier Science Publishing Company, 1992.

[2] Briggs, W. L. A Multigrid Tutorial. SIAM, 1987.

[3] Collier, J. R., Monk, N. A. M., Maini, P. K., And Lewis, J. H. Pattern formation by lateral inhibition with feedback: A mathematical model of delta-notch intercellular signalling. $J$. Theor. Biol. 183 (1996), 429-446.

[4] Dab, D., Lawniczak, A., Boon, J.-P., And Kapral, R. Cellular-automaton models for reactive systems. Phys. Rev. Lett. 64, 20 (1990), 2462-2465.

[5] Dallon, J. C., And Othmer, H. G. A discrete cell model with adaptive signalling for aggregation of dictyostelium discoideum. Phil. Trans. R. Soc. Lond. B 352, 1357 (1997), 391-417.

[6] Dallon, J. C., Sherratt, J. A., And Maini, P. K. Mathematical modelling of extracellular matrix dynamics using discrete cells: Fiber orientation and tissue regeneration. J. Theor. Biol. (1999). To appear.

[7] Durrett, R., And Levin, S. The importance of being discrete (and spatial). Theor. Popul. Biol. 46 (1994), 363-394.

[8] Edelstein-Keshet, L., and Ermentrout, B. G. Models for contact-mediated pattern formation: cells that form parallel arrays. J. Math. Biol. 29 (1990), 33-58. 
[9] Flach, S., and Willis, C. R. Discrete breathers. Phys. Rep. 295, 5 (March 1998), 181-264.

[10] Ghosh, T. K., Peng, H., And Bankslee, P. Analysis of fabric deformation in a roll-making operation. Text. Res. J. 62, 11 (1992), 669-676.

[11] Gibbons, M. R., And Hewett, D. W. Characterization of the darwin direct implicit particlein-cell mehod and resulting guidelines for operation. J. Comp. Phys. 130, 1 (1997), 54-66.

[12] Hockney, R. W., and Eastwood, J. W. Computer Simulations Using Particles. Institute of Physics Publishing, 1988.

[13] Höfer, T., Sherratt, J. A., And Maini, P. K. Dictyostelium discoideum: cellular selforganization in an excitable biological medium. Proc. R. Soc. Lond. B 259 (1995), 249-257.

[14] Hundsdorfer, W. H., And Verwer, J. G. Stability and convergence of the Peaceman-Rachford ADI method for initial-boundary value problems. Math. Comp. 53, 187 (1989), 81-101.

[15] Keener, J. P. Principles of Applied Mathematics Transformation and Approximation. AddisonWesley, 1988.

[16] Lewis, M. A., White, K. A. J., And Murray, J. D. Analysis of a model for wolf territories. J. Math. Biol. 35, 7 (1997), 749-774.

[17] Olsen, L., Maini, P. K., Sherratt, J. A., and Dallon, J. C. Mathematical modelling of anisotropy in fibrous connective tissue. Math. Biosci. 158 (1999), 145-170.

[18] O'Rourke, P. J., And Brackbill, J. U. On particle-grid interpolation and calculating chemistry in particle-in-cell methods. J. Comp. Phys. 109 (1993), 37-52.

[19] Owen, M. R., And Sherratt, Jonathan, A. Mathematical modelling of juxtacrine cell signalling. Math. Biosci. 152 (1998), 125-150.

[20] Peskin, Charles, S., And McQueen, D. M. Fluid dynamics of the heart and its valves. In Case Studies in mathematical Modeling Ecology, Physiology, and Cell Biology, H. G. Othmer, F. R. Adler, M. A. Lewis, and J. C. Dallon, Eds. Prentice Hall, 1997.

[21] Ralston, A., And Rabinowitz, P. A First Course in Numerical Analysis. McGraw Hill Book Company, New York, 1978. 
[22] Richtymer, R. D., And Morton, K. W. Difference Methods for Initial Value Problems. John Wiley, New York, 1967.

[23] Savill, N. J., And Hogeweg, P. Modelling morphogenesis: From single cells to crawling slugs. J. Theor. Biol. 184 (1997), 229-235.

[24] Siu, C.-H., And Kamboj, R. K. Cell-cell adhesion and morphogenesis in Dictyostelium discoideum. Dev. Gen. 11 (1990), 277-387.

[25] Skulsky, D., Zhou, S. J., And Schreyer, H. L. Application of a particle-in-cell method to solid mechanics. Comp. Phys. Commun 87, 1-2 (1995), 236-252.

[26] Smith, G. D. Numerical Solution of Partial Differential Equations: Finite Difference Methods. Oxford University Press, 1985.

[27] Tang, Y., and Othmer, H. G. Excitation, oscillations and wave propagation in a G-protein based model of signal transduction in Dictyostelium discoideum. Phil. Trans. R. Soc. Lond. B349 (1995), 179-195.

[28] Thacher, T., Ganesan, S., Askar, A., and Rabitz, H. A hybrid approach to modeling the dynamics of macromolecules. J. Chem. Phys. 85, 6 (1986), 3655-3673.

[29] Toda, M. Theory of nonlinear lattices, 2 ed., vol. 20 of Springer series in solid-state sciences. Springer, 1989.

[30] von Neumann, J. The general and logical theory of automata. In J. von Neumann, Collected Works, A. H. Taub, Ed., vol. 5. Pergamon Press, 1961, pp. 288-328.

[31] Wei, L. L., And Hudson, J. A. A coupled discrete continuum approach for modeling of waterflow in jointed rocks. Geotechnique 43, 1 (1993), 21-36.

[32] Weliky, M., And Oster, G. The mechanical basis of cell rearrangement i. epithelial morphogenesis during fundulus epiboly. Dev. 109 (1990), 373-386.

[33] Whitby, D. J., And Ferguson, M. W. J. The extracellular matrix of lip wounds in fetal, neonatal and adult mice. Dev. 112 (1991), 651-668.

[34] Wolfram, S. Theory and Applications of Cellular Automata. World Scientific, 1986. 